5. G. P. Johnson, Spaces of functions with values in a normed ring, Bull. Amer. Math. Soc. Abstract 62-4-458.

6. L. H. Loomis, An introduction to abstract harmonic analysis, New York, 1953.

7. G. W. Mackey, Commutative Banach algebras, edited by A. Blair, mimeographed notes, Harvard University, 1952.

8. L. Schwartz, Sur une propriété de synthèse spectrale dans les groupes non-compactes, C. R. Acad. Sci. Paris vol. 227 (1948) pp. 424-426.

9. A. B. Willcox, Note on certain group algebras, Proc. Amer. Math. Soc. vol. 7 (1956) pp. 874-879.

College of the City of New York and

YALE UNIVERSITY

\title{
A NOTE ON DERIVATIONS OF LIE ALGEBRAS II
}

\author{
G. F. LEGER
}

Let $L$ be a Lie algebra over a ground field of characteristic 0 and let $D(L)$ and $I(L)$ denote the Lie algebra of derivations and inner derivations of $L$ respectively.

The following theorem is proved, although not explicitly stated, in an earlier note. ${ }^{1}$

THEOREM 1. Let $L=S+R$ (Levi decomposition) and let $\mathfrak{A}(S)$ $=\{D \mid D \in D(L), D(S)=(0)\}$. Further let $\rho$ denote the restriction homomorphism of $D(L)$ into $D(R)$. Then $D(L)$ splits over $I(L) \rightleftarrows \rho(\mathfrak{A}(S))$ splits over $\rho(\mathfrak{A}(S)) \cap I(R)$.

If $S$ is a semi-simple Lie algebra and $M$ is any $S$-module then $M$ is the direct sum of $M^{S}$ and $S \cdot M$ where $M^{S}$ is the trivial submodule of $M$.

TheOREM 2. Let $L=S+R$ (Levi decomposition). If $R^{S} \subseteq Z(R)$ (the center of $R$ ) then $D(L)$ splits over $I(L) .^{2}$

Proof. Let $u \in R$ and suppose that the derivation of $R$ that is effected by $u$ is the restriction to $R$ of a derivation of $L$ that annihilates $S$. Then $[S, u] \subseteq Z(R)$. As an $S$-module, $R$ is the direct sum of $Z(R)$ and a complementary submodule $P$. The component of $u$ in $P$ is annihilated by $S$ so that $u \in R^{s}+Z(R) \subseteq Z(R)$. This means

Received by the editors May 17, 1958 and, in revised form, June 3, 1958.

1 Details will be found in G. F. Leger, $A$ note on the derivations of Lie algebras, Proc. Amer. Math. Soc. vol. 4 (1953). We refer to this note as I.

2 The author is grateful to the referee for putting Theorem 2 in this form. 
that the derivation of $R$ effected by $u$ is 0 . Now it follows from Theorem 1 that $D(L)$ splits over $I(L)$.

At the end of $I$ is the following example (Hochschild's) of a nilpotent Lie algebra $R$ such that $D(R)$ does not split over $I(R) . R$ has a basis $\left(x_{1}, x_{2}, x_{3}, x_{4}\right)$ over a ground field $K$ (of characteristic 0 ) where $\left(x_{2}, x_{3}, x_{4}\right)$ is abelian and $\left[x_{1}, x_{2}\right]=x_{4},\left[x_{1}, x_{3}\right]=0,\left[x_{1}, x_{4}\right]=0$. Now let $L=S+R$ where $S$ has a basis $\left(s_{1}, s_{2}, s_{3}\right)$ over $K$ with $\left[s_{i}, x_{j}\right]$ $=0$, for $i=1,2,3$ and $j=3,4$,

$$
\begin{array}{llll}
{\left[s_{1}, s_{2}\right]=2 s_{2},} & {\left[s_{2}, s_{3}\right]=s_{1},} & {\left[s_{2}, x_{2}\right]=x_{1},} \\
{\left[s_{1}, s_{3}\right]=-2 s_{3},} & {\left[s_{1}, x_{2}\right]=-x_{2},} & {\left[s_{3}, x_{1}\right]=x_{2},} \\
{\left[s_{1}, x_{1}\right]=x_{1},} & {\left[s_{2}, x_{1}\right]=0,} & {\left[s_{3}, x_{2}\right]=0 .}
\end{array}
$$

Then $S$ is simple and $L=S+R$ is a Levi decomposition of $L$. Further $R^{S} \subseteq Z(R)$ so that $D(L)$ splits over $I(L)$ by Theorem 2 . Thus we have an example of a Lie algebra $L$ with radical $R$ such that $D(L)$ splits over $I(L)$ but $D(R)$ does not split over $I(R)$.

This settles a question left open in I.

University of Pittsburgh 